\title{
Impact of metal pollution on shrimp Crangon affinis by NMR-based metabolomics
}

\author{
Chenglong Ji ${ }^{\mathrm{a}, 1}$, Deliang Yu ${ }^{\mathrm{a}, \mathrm{b}, 1}$, Qing Wang ${ }^{\mathrm{a}}$, Fei $\mathrm{Li}^{\mathrm{a}}$, Jianmin Zhao ${ }^{\mathrm{a}}$, Huifeng $\mathrm{Wu}^{\mathrm{a}, *}$

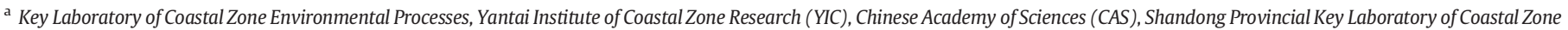 \\ Environmental Processes, YICCAS, Yantai 264003, PR China \\ ${ }^{\mathrm{b}}$ University of Chinese Academy of Sciences, Beijing 100049, PR China
}

\section{A R T I C L E I N F O}

\section{Article history:}

Received 17 January 2016

Received in revised form 15 February 2016

Accepted 19 February 2016

Available online xxxx

\section{Keywords:}

Metal pollution

Crangon affinis

Metabolomics

${ }^{1}$ H NMR

\begin{abstract}
A B S T R A C T
Both cadmium and arsenic are the important metal/metalloid pollutants in the Bohai Sea. In this work, we sampled the dominant species, shrimp Crangon affinis, from three sites, the Middle of the Bohai Sea (MBS), the Yellow River Estuary (YRE) and the Laizhou Bay (LZB) along the Bohai Sea. The concentrations of metals/metalloids in shrimps $C$. affinis indicated that the YRE site was polluted by $\mathrm{Cd}$ and $\mathrm{Pb}$, while the LZB site was contaminated by As. The metabolic differences between shrimps $C$. affinis from the reference site (MBS) and metal-pollution sites (YRE and LZB) were characterized using NMR-based metabolomics. Results indicated that the metal pollutions in YRE and LZB induced disturbances in osmotic regulation and energy metabolism via different metabolic pathways. In addition, a combination of alanine and arginine might be the biomarker of $\mathrm{Cd}$ contamination, while BCAAs and tyrosine could be the biomarkers of arsenic contamination in C. affinis.
\end{abstract}

(c) 2015 Elsevier Ltd. All rights reserved.
With the rapid development of industry, metal pollution has become a serious environmental problem in the marine and coastal environments in the Bohai Sea. Mu et al. (2009) reported that cadmium (Cd) and arsenic (As) were the dominant metal/metalloid pollutants in the sediments from the Laizhou Bay. In the cultured shellfishes collected from the Laizhou Bay, an early report showed that the samples from more than $50 \%$ of the sampling sites were polluted by $\mathrm{Cd}$ (Liu et al., 2004). Due to the discharge of industrial effluents along the Yellow River, the Yellow River Estuary has also become a metal-polluted site along the Bohai coast. Since the accumulated metals can induce toxicities in marine organisms, the great concern has been raised over the health risks of metal pollutions to aquatic organisms.

The marine shrimp Crangon affinis is the dominant species and widely distributed in the Yellow Sea and the Bohai Sea (Cheng, 2005). C. affinis plays a very important role in the food chain since this species is the main bait of fishes. Hence, they can maintain the marine ecosystem health (Cheng, 2005). In addition, shrimp C. affinis is consumed as delicious seafood by local residents (Xu et al., 2008). Therefore, it is necessary to investigate the biological effects of metal pollutions to this shrimp species. To our knowledge, however, few studies have been focused on the metal pollution-induced biological effects in shrimp C. affinis.

In recent years, the-omics techniques, such as genomics, transcriptomics, proteomics and metabolomics, have been widely used in environmental sciences to elucidate the biological effects induced by environmental stressors to organisms (Ji et al., 2013; Santos et al.,

\footnotetext{
* Corresponding author.

E-mail address: hfwu@yic.ac.cn (H. Wu).

1 These two authors contributed equally to this work.
}

2010; Williams et al., 2009). Metabolomics is a newly establishedomics technique that focuses on all the small molecular metabolites ( $<1000 \mathrm{Da}$ ) in organisms (Jones et al., 2008; Viant et al., 2003). A comparative analysis on the metabolome profiles in organisms under environmental stress can provide metabolic responses induced by environmental stressors (Fasulo et al., 2012; Kwon et al., 2012; Cappello et al., 2013). In a previous study, Kwon et al. (2012) successfully applied NMR-based metabolomics to investigate the biological effects of metal pollution in marine mussels (Mytilus edulis) sampled from a metal-polluted area (Onsan Bay). Their work confirmed the applicability of NMR-based metabolomics to characterize metal pollutioninduced biological effects in organisms.

In this study, the NMR-based metabolomics was used to investigate metabolic responses in shrimp C. affinis to metal pollutions. The shrimps C. affinis were collected from three sites in the Yellow River Estuary, the Laizhou Bay and the Middle of the Bohai Sea, respectively. Among these sampling sites, the former two sites might be contaminated by metals or metalloids (such as $\mathrm{Cd}, \mathrm{Pb}$ and As), respectively, and the site in the Middle of the Bohai Sea was a relatively clean and therefore used as the reference. The muscle tissues of individual shrimps C. affinis were examined for the metabolic profiles. The aims of this study were to characterize the biological effects in shrimps $C$. affinis exposed to metal pollutions using NMR-based metabolomics.

The shrimps $C$. affinis were collected from the Yellow River Estuary (YRE, $37^{\circ} 45^{\prime} 0^{\prime \prime} \mathrm{N}, 119^{\circ} 15^{\prime} 0^{\prime \prime} \mathrm{E}$ ), the Laizhou Bay (LZB, $39^{\circ} 0^{\prime} 0^{\prime \prime} \mathrm{N}$, $120^{\circ} 30^{\prime} 0^{\prime \prime} \mathrm{E}$ ) and the Middle of the Bohai Sea (MBS, $37^{\circ} 15^{\prime} 0^{\prime \prime} \mathrm{N}$, $119^{\circ} 30^{\prime} 0^{\prime \prime}$ E) along the Bohai Sea in May, 2015 (Fig. 1). Six individual shrimps with similar sizes were sampled from each site, and the digestive gland and muscle tissues were immediately dissected and flash frozen in liquid $\mathrm{N}_{2}$. After transported to our laboratory, these shrimp 


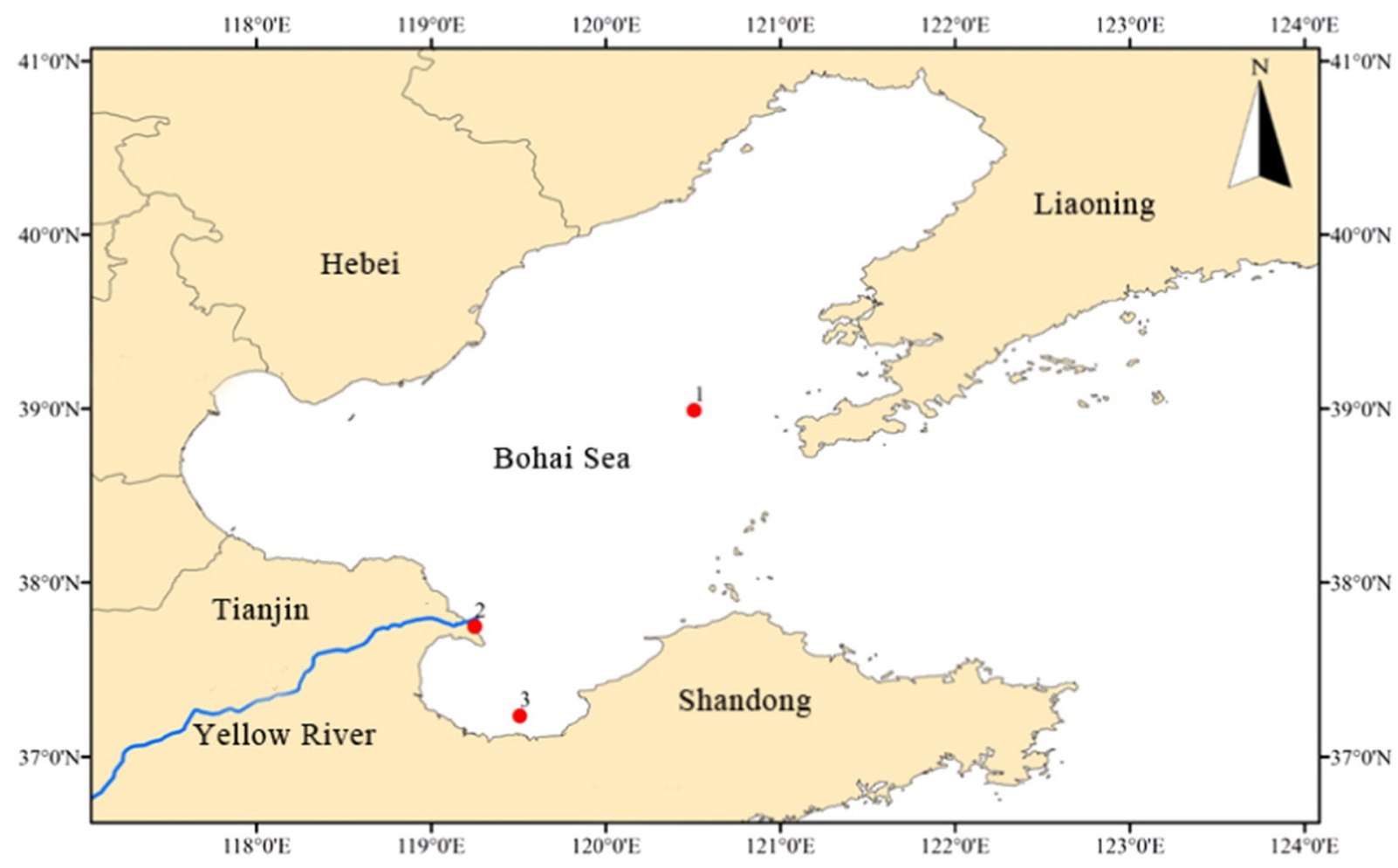

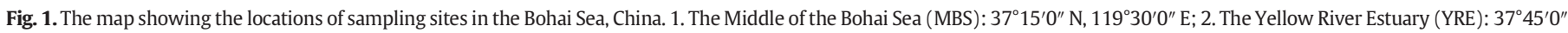
$\mathrm{N}, 119^{\circ} 15^{\prime} 0^{\prime \prime} \mathrm{E}$; 3 . The Laizhou Bay (LZB): $39^{\circ} 0^{\prime} 0^{\prime \prime} \mathrm{N}, 120^{\circ} 30^{\prime} 0^{\prime \prime} \mathrm{E}$.

samples were stored at $-80^{\circ} \mathrm{C}$ before metabolite extraction and metal analysis. All the practical procedures for shrimp sampling were strictly performed according to the guidelines suggested by Hines et al. (2007) and Vidal-Liñán and Bellas (2013).

Polar metabolites were extracted from the muscle tissues of shrimps ( $n=6$ ) by a modified extraction protocol using methanol/chloroform (Zhang et al., 2011). Briefly, the muscle tissue $(\sim 60 \mathrm{mg})$ was homogenized using a high throughput homogenizer (Precellys 24, Bertin Technologies, France) and extracted in $4 \mathrm{~mL} / \mathrm{g}$ of methanol, $0.85 \mathrm{~mL} / \mathrm{g}$ of water and $2 \mathrm{~mL} / \mathrm{g}$ of chloroform. The mixture was shaken and centrifuged ( $5 \mathrm{~min}, 3000 \mathrm{~g}$, at $4{ }^{\circ} \mathrm{C}$ ), and the supernatant substance was removed. A total of $2 \mathrm{~mL} / \mathrm{g}$ of chloroform and $2 \mathrm{~mL} / \mathrm{g}$ of water were added to the supernatant, and the mixture was vortexed and then centrifuged again $\left(10 \mathrm{~min}, 3000 \mathrm{~g}, 4^{\circ} \mathrm{C}\right)$. The methanol/water layer with polar metabolites was transferred to a glass vial. The sample was dried in a centrifugal concentrator and stored at $-80{ }^{\circ} \mathrm{C}$ before NMR measurement. It was subsequently re-suspended in $600 \mu \mathrm{L}$ of $100 \mathrm{mM}$ phosphate buffer $\left(\mathrm{Na}_{2} \mathrm{HPO}_{4}\right.$ and $\mathrm{NaH}_{2} \mathrm{PO}_{4}$ with $0.5 \mathrm{mM} \mathrm{TSP}, \mathrm{pH}$ 7.0) in $\mathrm{D}_{2} \mathrm{O}$. The mixture was vortexed and then centrifuged at $3000 \mathrm{~g}$ for $5 \mathrm{~min}$ at $4{ }^{\circ} \mathrm{C}$. The supernatant substance $(550 \mu \mathrm{L})$ was then pipetted into a $5 \mathrm{~mm}$ NMR tube for NMR analysis.

Extracts of shrimp muscle samples were analyzed on a Bruker AV 500 NMR spectrometer performed at $500.18 \mathrm{MHz}$ (at $298 \mathrm{~K}$ ) (Zhang et al., 2011). One-dimensional (1-D) ${ }^{1} \mathrm{H}$ NMR spectra were obtained using a $11.9 \mu$ s pulse, $6009.6 \mathrm{~Hz}$ spectral width, mixing time $0.1 \mathrm{~s}$, and $3.0 \mathrm{~s}$ relaxation delay with standard 1D NOESY pulse sequence, with 128 transients collected into 16384 data points. Datasets were then zero-filled to 32768 points, and exponential line-broadenings of $0.3 \mathrm{~Hz}$ were applied before Fourier transformation. All ${ }^{1} \mathrm{H}$ NMR spectra were phased, baseline-corrected, and calibrated (TSP at $0.0 \mathrm{ppm}$ ) manually using TopSpin (version 2.1, Bruker).

All one dimensional ${ }^{1} \mathrm{H}$ NMR spectra were converted to a data matrix using the custom-written ProMetab software in Matlab (V7.0, The MathWorks, Natick, MA, USA) (Viant et al., 2003). Each spectrum was segmented into bins with a width of $0.005 \mathrm{ppm}$ between 0.2 and
$10.0 \mathrm{ppm}$. The bins of residual water peak between 4.70 and $5.20 \mathrm{ppm}$ were excluded from all the ${ }^{1} \mathrm{H}$ NMR spectra. The total spectral area of the remaining bins was normalized to unity to facilitate the comparison between the spectra. All the NMR spectra were generalized log transformed with a transformation parameter $\lambda=2.0 \times 10^{-9}$ to stabilize the variance across the spectral bins and to increase the weightings of the less intense peaks (Zhang et al., 2011). Data were mean-centered before multivariate data analysis.

The unsupervised pattern recognition method, principal component analysis (PCA) was used to reduce the dimensionality of the data and separate the groups of shrimp samples from MBS, YRE and LZB. Oneway analysis of variance (ANOVA) was conducted on the PC scores from each group to test the statistical significance $(P<0.05)$ of separations. Furthermore, the supervised multivariate data analysis methods, partial least squares discriminant analysis (PLS-DA) and orthogonal projection to latent structure with discriminant analysis (O-PLS-DA), were sequentially carried out to uncover and extract the statistically significant metabolite variations related to metal pollutions. The results were visualized in terms of score plots to show the classifications and corresponding loading plots to show the NMR spectral variables contributing to the classifications. The model coefficients were calculated from the coefficients incorporating the weight of the variables in order to enhance interpretability of the model. Then metabolic differences responsible for the classifications between the reference (MBS) and the metal pollution-exposed group (YRE or LZB) could be detected in the coefficient-coded loadings plot generated by using MATLAB (V7.0, the Mathworks Inc., Natwick, USA) with an in-house developed program and was color-coded with absolute value of coefficients (r). A hot color (i.e., red) corresponds to the metabolites with highly positive/negative significances in discriminating between groups, while a cool color (i.e., blue) corresponds to no significance. The correlation coefficient was determined on the basis of the test for the significance of the Pearson's product-moment correlation coefficient. The validation of the model was conducted using 6 -fold cross validation and the crossvalidation parameter $Q^{2}$ was calculated, and an additional validation 
method, permutation test (permutation number $=200$ ), was also conducted in order to evaluate the validity of the PLS-DA models. The $\mathrm{R}^{2}$ in the permutated plot described how well the data fit the derived model, whereas $Q^{2}$ value describes the predictive ability of the constructed model and provides a measure of the model quality. If the maximum value of $Q^{2}$ max from the permutation test was smaller than or equal to the $Q^{2}$ of the real model, the model was regarded as a predictable model. Similarly, the $R^{2}$ value and difference between the $R^{2}$ and $Q^{2}$ were used to evaluate the possibility of over-fitted models (Feng et al., 2013). Metabolites were assigned following the tabulated chemical shifts and by using the software, Chenomx (Evaluation Version, Chenomx Inc., Edmonton, Alberta, Canada).

Each digestive gland tissue sample from the shrimps $(n=6)$ was dried to the constant weight and then digested thoroughly in concentrated $\mathrm{HNO}_{3}$ at $80{ }^{\circ} \mathrm{C}$ for $12 \mathrm{~h}$. Metal concentration in the shrimp samples was determined by inductively coupled plasma mass spectrometry (ICP-MS, Agilent $7700 \times$ ). Appropriate internal standards (Sc, $\mathrm{Ge}, \mathrm{In}, \mathrm{Bi}$ ) were selected to correct the sensitivity drift and matrix effect. A quality control sample was repeatedly measured after every 10 samples. The recovery of the analyzed metals from the standard reference material (SRM 1566b, oyster tissue) was within 10\% deviation from the certified values except $\mathrm{Cr}$, of which the certified concentration was not available. Metal concentrations were expressed as mean \pm standard deviation (S.D.). All the data of metal concentrations were subjected to principal component analysis with autoscaling and Student $t$-test. A P value less than 0.05 was considered significant. Statistical analysis was performed using Minitab software (Version 15, Minitab Inc. PA, USA).

Due to the lower capability of metal accumulation than marine bivalves such as mussels, oysters and clams, shrimp C. affinis is not a member of the bioindicators in 'Mussel Watch Program' (Goldberg et al., 1983). However, shrimp C. affinis is the dominant species in the Bohai Sea ecosystem and plays a vital role in the food chain (Cheng, 2005). Therefore it is necessary to elucidate the biological responses induced by metal pollutions in this species. The average concentrations $(\mu \mathrm{g} / \mathrm{g}$ wet weight) of nine metals ( $\mathrm{Cr}, \mathrm{Mn}, \mathrm{Ni}, \mathrm{Co}, \mathrm{Fe}, \mathrm{Cu}, \mathrm{Zn}, \mathrm{Cd}$ and $\mathrm{Pb}$ ) and two metalloids (As and Se) in the shrimps $C$. affinis collected from the reference (MBS) and metal contaminated sites (YRE and LZB) are indicated in Table 1. Principal component analysis (PCA) was conducted to summarize the differences of metal/metalloid concentrations from different sampling sites. The biplot with shrimp samples from the sampling sites (MBS, YRE and LZB) and variables (nine metals and two metalloids) is shown in Fig. 2, with the first two principal components (PCs) containing $52.26 \%$ of the variance from the original eleven dimensions. As shown in the biplot (Fig. 2), these three groups of samples from MBS, YRE and LZB were basically separated along PC1 or PC2 axis.

Table 1

Metal/metalloid concentrations in the digestive gland tissues from shrimps Crangon affinis sampled from three sampling sites, the middle of the Bohai Sea (MBS), the Yellow River Estuary (YRE) and the Laizhou Bay (LZB).

\begin{tabular}{|c|c|c|c|}
\hline \multirow{2}{*}{$\begin{array}{l}\text { Metal/metalloid } \\
\text { concentration }^{\mathrm{a}}\end{array}$} & \multicolumn{3}{|l|}{ Sampling site } \\
\hline & MBS & YRE & LZB \\
\hline $\mathrm{Cr}$ & $0.29 \pm 0.07$ & $0.36 \pm 0.17$ & $0.24 \pm 0.07$ \\
\hline Mn & $9.64 \pm 2.95$ & $7.26 \pm 1.79$ & $6.51 \pm 1.87$ \\
\hline $\mathrm{Ni}$ & $0.83 \pm 0.17$ & $0.54 \pm 0.07$ & $0.55 \pm 0.18$ \\
\hline Co & $0.19 \pm 0.05$ & $0.22 \pm 0.11$ & $0.18 \pm 0.05$ \\
\hline $\mathrm{Fe}$ & $1.68 \pm 0.53$ & $1.65 \pm 0.40$ & $1.58 \pm 0.54$ \\
\hline $\mathrm{Cu}$ & $62.17 \pm 17.81$ & $75.84 \pm 23.78$ & $68.02 \pm 10.61$ \\
\hline $\mathrm{Zn}$ & $33.58 \pm 8.47$ & $29.03 \pm 1.92$ & $30.81 \pm 6.69$ \\
\hline As & $22.00 \pm 8.11$ & $9.78 \pm 4.50$ & $92.11 \pm 55.39^{*}$ \\
\hline $\mathrm{Se}$ & $4.13 \pm 0.91$ & $2.54 \pm 0.42^{*}$ & $3.22 \pm 1.26$ \\
\hline $\mathrm{Cd}$ & $1.54 \pm 0.49$ & $3.42 \pm 0.87^{*}$ & $2.33 \pm 1.30$ \\
\hline $\mathrm{Pb}$ & $0.19 \pm 0.04$ & $0.31 \pm 0.09^{*}$ & $0.22 \pm 0.06$ \\
\hline
\end{tabular}

* $(P<0.05)$ means the significant differences of metal concentrations between clean (MBS) and metal pollution sites (YRE or LZB) (Student $t$-test).

a Data are shown as mean \pm standard deviation $(n=6)$. Values are presented as $\mu \mathrm{g} / \mathrm{g}$ wet weight.

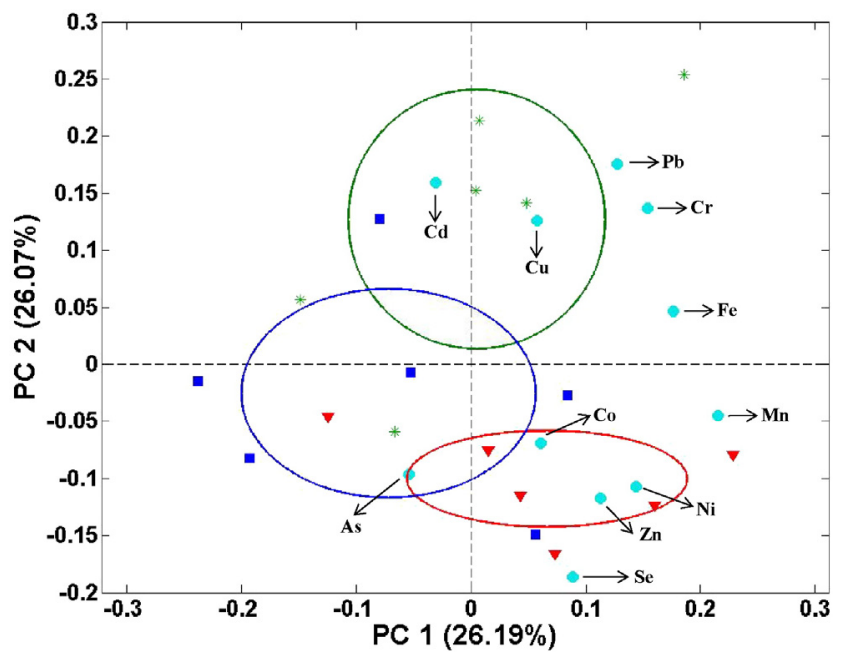

Fig. 2. The biplot containing PC scores of shrimp samples from three sampling sites, MBS $(\boldsymbol{\nabla})$, YRE $\left(^{*}\right)$ and LZB ( $\left.\boldsymbol{\square}\right)$ and variable (eleven metals/metalloid) contributions for the clustering of shrimp samples. Ellipses represent mean \pm standard deviation for each group of samples from different sites.

Overall, the shrimp samples from YRE showed significantly higher tissue concentrations of $\mathrm{Cd}$ and $\mathrm{Pb}$ than those in the samples from MBS. Clearly, the shrimp samples from LZB site accumulated the highest level of As (Table 1 and Fig. 2). As reported previously, both Cd and As were the main metal/metalloid pollutants in the Bohai Sea environment (Mu, 2009), which was similarly observed in this work as shown by the concentrations of $\mathrm{Cd}$ and As in shrimps C. affinis.

The representative ${ }^{1} \mathrm{H}$ NMR spectra of muscle tissue extracts of shrimps sampled from MBS, YRE and LZB sites are shown in Fig. 3. A total of 33 metabolites were identified, including amino acids (valine, leucine, isoleucine, alanine, arginine, glutamate, glutamine, proline, glycine, etc.), osmolytes (betaine, taurine, homarine, myo-inositol and hypotaurine), energy storage compounds (glucose, glycogen and ATP) and organic acids (lactate, succinate and fumurate). Apparently, the ${ }^{1} \mathrm{H}$ NMR spectra were dominated by the organic osmolyte, betaine which plays an important role in the osmotic regulation in marine invertebrates (Fig. 3).

PCA was performed on the ${ }^{1} \mathrm{H}$ NMR spectral data to summarize the similarities and differences between the shrimp groups from the sampling sites. The PC scores plot presented significant $(P<0.05)$ separations between reference (MBS) and metal pollution-exposed groups (YRE and LZB). In details, the shrimp group from MBS site is located along positive PC2 (14.57\% of variation) axis (Fig. 4). Both YRE and LZB groups are separated along negative PC2 axis. In addition, the YRE and LZB groups are clearly separated along positive and negative PC1 axis (58.41\% of variation), respectively. These significant separations between three groups of shrimp samples demonstrated the significant metabolic differences in shrimps sampled from these sampling sites, MBS, YRE and LZB. To discover the metabolic responses, O-PLS-DA was conducted on the ${ }^{1} \mathrm{H}$ NMR spectral data from the reference (MBS) and metal pollution-exposed shrimp groups (YRE and LZB), respectively (Fig. 5). The score plots (Fig. 5A and C) generated from O-PLS-DA indicated clear classifications between the reference (MBS) and metal pollution-exposed groups (YRE and LZB), with reliable $Q^{2}$ values $(>0.4)$. The significant metabolic responses induced by metal pollutions of these sampling sites (YRE and LZB) were labeled in the corresponding loading plots of O-PLS-DA (Fig. 5B and D).

As shown in the loading plot of O-PLS-DA (Fig. 5B), the shrimp samples from YRE site had higher levels of alanine, arginine, glutamine and AMP and lower levels of malonate, phosphocholine, glycine and homarine, than those in the shrimp samples from the reference site, MBS. The metabolic profiles in the shrimp samples from LZB site presented higher concentrations of arginine, glycine, ATP and AMP and 


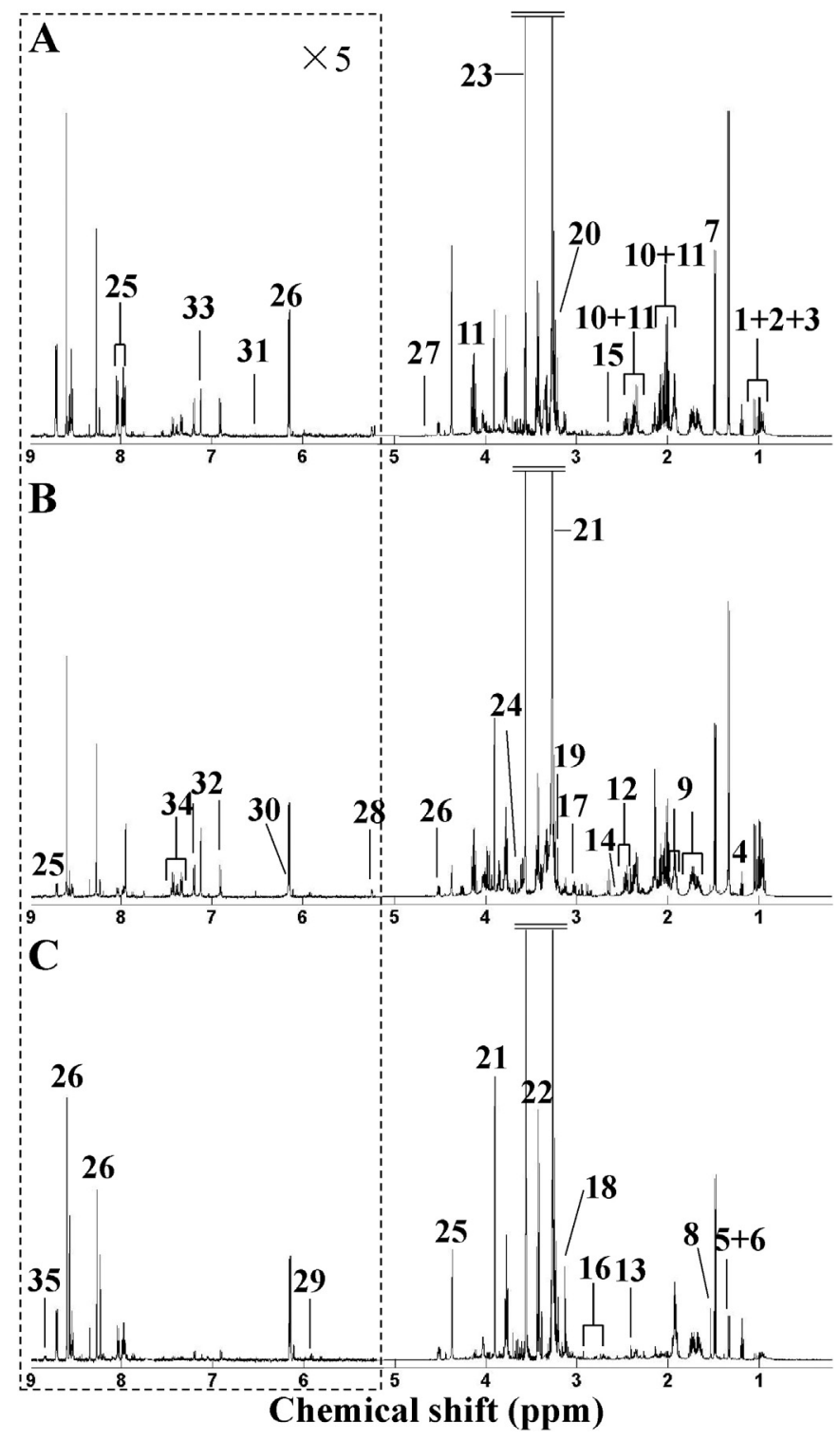

Fig. 3. Representative 1-dimensional $500 \mathrm{MHz}{ }^{1} \mathrm{H}$ NMR spectra of the muscle tissue extracts from shrimps $C$. affinis from the reference site (MBS, A) and metal polluted sites (YRE and LZB, B and C). Keys: (1) leucine, (2) isoleucine, (3) valine, (4) ethanol, (5) lactate, (6) threonine, (7) alanine, (8) unknown 1 (1.54 ppm), (9) arginine, (10) glutamate, (11) proline, (12) glutamine, (13) succinate, (14) $\beta$-alanine, (15) hypotaurine, (15) asparagine, (17) lysine, (18) malonate, (19) choline, (20) phosphocholine, (21) betaine, (22) taurine, (23) glycine, (24) myo-inositol, (25) homarine, (26) AMP, (27) $\beta$-glucose, (28) $\alpha$-glucose, (29) glycogen, (30) unknown 2 (5.96 ppm), (30) ATP, (31) fumarate, (32) tyrosine, (33) histidine, (34) phenylalanine and (35) $\mathrm{NAD}^{+}$.

lower concentrations of branched chain amino acids (BCAAs: valine, leucine and isoleucine) lactate, proline, myo-inositol, homarine, glucose, tyrosine, histidine and phenylalanine (Fig. 5D). The metabolic responses including arginine, AMP and homarine were similarly altered in the shrimp samples from YRE and LZB sites.

Free amino acids are usually involved in both osmotic regulation and energy metabolism in marine invertebrates (Viant et al., 2003). In the shrimp samples from YRE site, several amino acids including alanine, arginine and glutamine were of higher abundance than those in the shrimp samples from the reference site (MBS), which suggested the disturbance in osmotic regulation induced by metal pollution in shrimps. As known osmolytes, however, both glycine and homarine were of relatively low abundances compared with those shrimp samples from the MBS site. It seemed that these two metabolites were decreased to

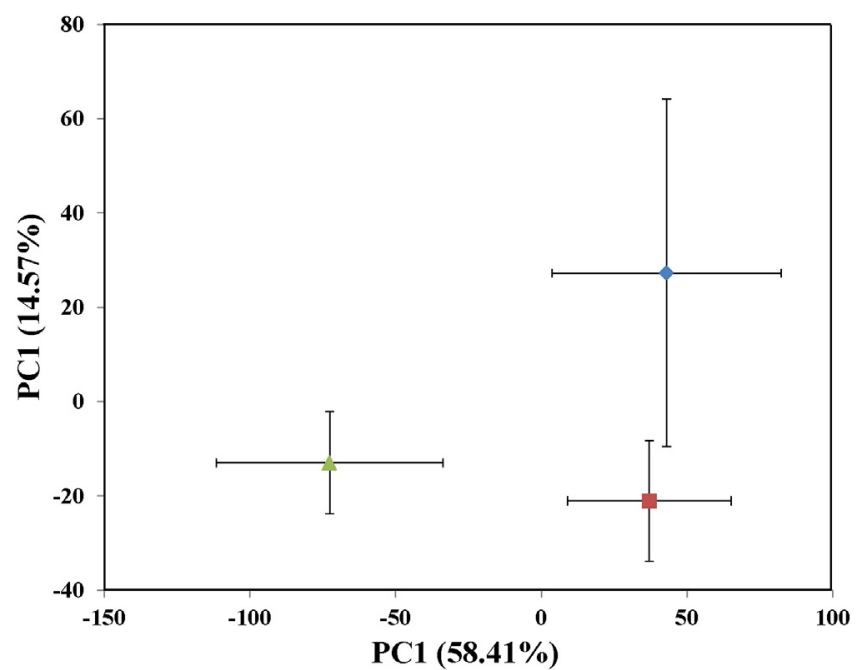

Fig. 4. Mean principal component analysis (PCA) scores plot of $P C 1$ versus $P C 2$ for ${ }^{1} H$ NMR spectra of the muscle tissue extracts from shrimps $C$. affinis sampled from $\operatorname{MBS}(\boldsymbol{\nabla})$, YRE $(\boldsymbol{\square})$ and LZB $(\boldsymbol{\Delta})$. The classes were presented as mean \pm standard deviation of PC scores for each group of shrimp samples.

compensate for the increased amino acids, alanine, arginine and glutamine to regulate the osmotic balance in shrimps. Phosphocholine and ADP are involved in the conversion to choline and ATP. Malonate is a competitive inhibitor of the enzyme succinate dehydrogenase that is also related to energy metabolism. AMP is the product in ATP hydrolysis. The altered AMP, phosphocholine and malonate implied the disturbance in energy metabolism caused by metal pollution in shrimps from the YRE site. In the previous study, both alanine and arginine were elevated in Cd-exposed clam Ruditapes philippinarum (Wu et al., 2013a), which is consistent with the alteration of alanine and arginine in shrimps from YRE site. Interestingly, $\mathrm{Cd}$ is the main metal contaminant in the shrimp from YRE site. Therefore, a combination of alanine and arginine might be the biomarker of $\mathrm{Cd}$ contamination in shrimp C. affinis. In the shrimp samples from LZB site, the altered amino acids and organic osmolytes, including BCAAs, arginine, glycine, proline, tyrosine, histidine, phenylalanine, myo-inositol and homarine, clearly indicated the disturbance in osmotic regulation. The elevated ATP and AMP and the depleted glucose demonstrated the enhanced energy consumption to deal with the stress induced by metal pollution in these shrimps from LZB site. In addition, the depleted lactate displayed the reduced anaerobiosis in the shrimps from LZB site. In arsenate-exposed clam $R$. philippinarum, the similar alteration of BCAAs and tyrosine was also observed (Wu et al., 2013b). Since the shrimp samples from LZB site were contaminated by arsenic, these amino acids could be used as biomarkers of arsenic contamination in shrimp C. affinis.

The concentrations of metals/metalloids in shrimps $C$. affinis indicated that the YRE site was mainly polluted by $\mathrm{Cd}$ and $\mathrm{Pb}$, while the LZB site was contaminated by As. In this study, the metabolic differences between shrimps $C$. affinis from the reference site (MBS) and metalpollution sites (YRE and LZB) were characterized using NMR-based metabolomics. Results indicated that the metal pollutions in YRE and LZB induced disturbances in osmotic regulation and energy metabolism via different metabolic pathways marked by the different metabolic biomarkers compared with those shrimp samples from the reference site, MBS. In addition, a combination of alanine and arginine might be the biomarker of $\mathrm{Cd}$ contamination in shrimp $C$. affinis. The amino acids including BCAAs and tyrosine could be used as biomarkers for arsenic contamination in shrimp C. affinis. This study demonstrates that metabolomics is useful to elucidate the metabolic responses and biological effects induced by metal pollutions. 

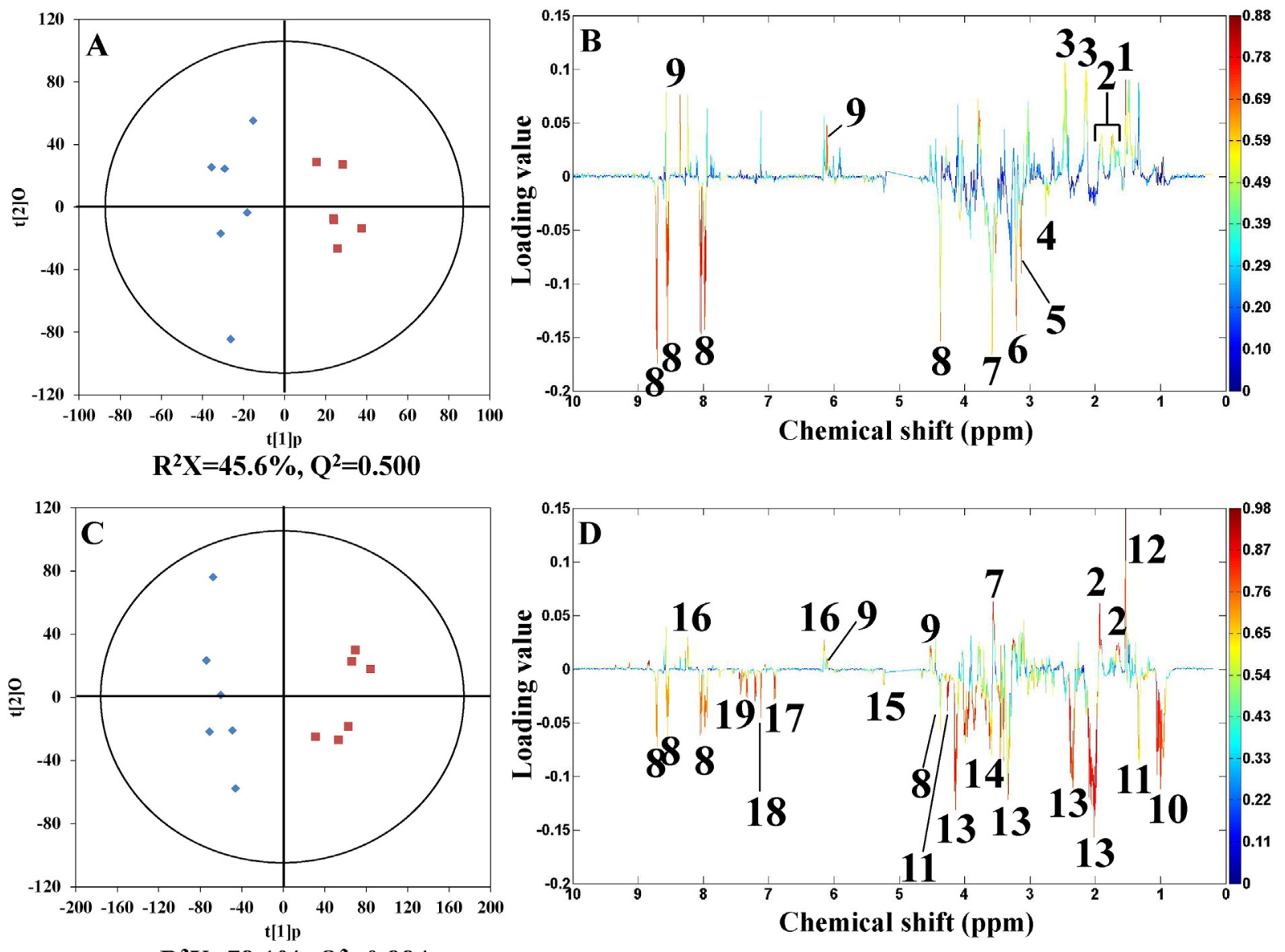

$\mathrm{R}^{2} \mathrm{X}=\mathbf{7 8 . 1 \%}, \mathrm{Q}^{2}=\mathbf{0 . 8 8 4}$

Fig. 5. OPLS-DA scores derived from ${ }^{1} \mathrm{H}$ NMR spectra of the muscle tissue extracts from shrimps sampled from reference site ( $)$ and metal polluted sites ( $\left.\mathbf{\square}\right)$, (A) MBS vs. YRE and (C) MBS vs. LZB and corresponding coefficient plots (B) and (D). The color map shows the significance of metabolite variations between the two classes (reference and metal polluted sites). Peaks in the positive direction indicate metabolites that are more abundant in the metal polluted groups. Consequently, metabolites that are more abundant in the samples from reference site are presented as peaks in the negative direction. Keys: (1) alanine, (2) arginine, (3) glutamine, (8) unknown 1 (2.76 ppm), (5) malonate, (6) phosphocholine, (7) glycine, (8) homarine, (9) AMP, (10) branched chain amino acids: valine, leucine and isoleucine, (11) lactate, (12) unknown 2 (1.54 ppm), (13) proline, (14) myo-inositol, (15) glucose, (16) ATP, (17) tyrosine, (18) histidine and (19) phenylalanine.

\section{Acknowledgments}

This work was supported by a Key Project from National Key Basic Research Program of China (2015CB453303) and NSFC (21237004). We thank Prof. Mark Viant for the use of ProMetab software.

\section{References}

Cappello, T., Mauceri, A., Corsaro, C., Maisano, M., Parrino, V., Lo Paro, G., Messina, G., Fasulo, S., 2013. Impact of environmental pollution on caged mussels Mytilus galloprovincialis using NMR-based metabolomics. Mar. Pollut. Bull. 77, 132-139.

Cheng, J., 2005. Structure and diversity of invertebrate resources in the Yellow Sea (in Chinese). J. Fishery Sci. Chin. 12, 68-75.

Fasulo, S., Iacono, F., Cappello, T., Corsaro, C., Maisano, M., D'Agata, A., Giannetto, A., de Domenico, E., Parrino, V., Lo Paro, G., Mauceri, A., 2012. Metabolomic investigation of Mytilus galloprovincialis (Lamarck 1819) caged in aquatic environments. Ecotoxicol. Environ. Saf. 84, 139-146.

Feng, J., Li, J., Wu, H., Chen, Z., 2013. Metabolic responses of HeLa cells to silica nanoparticles by NMR-based metabolomic analyses. Metabolomics 9, 874-886.

Goldberg, E.D., Koide, M., Hodge, V., Flegal, A.R., Martin, J., 1983. United States mussel watch $-1977-1978$ results on trace metals and radionuclides. Estuar. Coast. Shelf Sci. 16, 69-93.

Hines, A., Oladiran, G.S., Bignell, J.P., Stentiford, G.D., Viant, M.R., 2007. Direct sampling of organisms from the field and knowledge of their phenotype: key recommendations for environmental metabolomics. Environ. Sci. Technol. 41, 3375-3381.

Ji, C., Wu, H., Wei, L., Zhao, J., Yu, J., 2013. Proteomic and metabolomic analysis reveal gender-specific responses of mussel Mytilus galloprovincialis to 2,2',4,4'tetrabromodiphenyl ether (BDE 47). Aquat. Toxicol. 140-141, 449-457.

Jones, O.A.H., Dondero, F., Viarengo, A., Griffin, J.L., 2008. Metabolic profiling of Mytilus galloprovincialis and its potential applications for pollution assessment. Mar. Ecol. Prog. Ser. 369, 169-179.
Kwon, Y.K., Jung, Y.S., Park, J.C., Seo, J., Choi, M.S., Hwang, G.S., 2012. Characterizing the effect of heavy metal contamination on marine mussels using metabolomics. Mar. Pollut. Bull. 64, 1874-1879.

Liu, F., Wang, H., Wang, D., 2004. Spatial distributions of heavy metals from the Laizhou Bay littoral wetland (in Chinese). Adv. Mar. Sci. 22, 486-492.

$\mathrm{Mu}, \mathrm{J.}$, 2009. The Evaluation of Ecological Environment for Key Fishery Waters in Bohai Sea (in Chinese) (Master's thesis) Ocean University of China.

Santos, E., Ball, J.S., Williams, T.D., Wu, H., Ortega, F., Van Aerle, R., Katsiadaki, I., Falciani, F., Viant, M.R., Chipman, J.K., Tyler, C.R., 2010. Identifying health impacts of exposure to copper using transcriptomics and metabolomics in a fish model. Environ. Sci. Technol. 44, 820-826.

Viant, M.R., Rosenblum, E.S., Tjeerdema, R.S., 2003. NMR-based metabolomics: a powerful approach for characterizing the effects of environmental stressors on organism health. Environ. Sci. Technol. 37, 4982-4989.

Vidal-Liñán, L., Bellas, J., 2013. Practical procedures for selected biomarkers in mussels, Mytilus galloprovincialis implications for marine pollution monitoring. Sci. Total Environ. 461-462, 56-64.

Williams, T.D., Wu, H., Santos, E., Ball, J., Katsiadaki, I., Brown, M.M., Baker, P., Ortega, F., Falciani, F., Craft, J.A., Tyler, C.R., Chipman, J.K., Viant, M.R., 2009. Hepatic transcriptomic and metabolomic responses in the stickleback (Gasterosteus aculeatus) exposed to environmentally relevant concentrations of dibenzanthracene. Environ. Sci. Technol. 43, 6341-6348.

Wu, H., Ji, C., Wang, Q., Liu, X., Zhao, J., Feng, J., 2013a. Manila clam Venerupis philippinarum as a biomonitor to metal pollution. Chin. J. Oceanol. Limnol. 31, 65-74.

Wu, H., Zhang, X., Wang, Q., Li, L., Ji, C., Liu, X., Zhao, J., Yin, X., 2013b. A metabolomics investigation on arsenic-induced toxicological effects in the clam Ruditapes philippinarum under different salinities. Ecotoxicol. Environ. Saf. 90, 1-6.

Xu, B., Chen, X., Tang, Z., Huang, H., Yu, Y., Zhang, X., Feng, C., 2008. Selectivity experiment on the cod-end mesh of beam shrimp trawl for catching Crangon affinis (in Chinese). Mar. Fisher. 30, 233-238.

Zhang, L., Liu, X., You, L., Zhou, D., Wang, O., Li, F., Cong, M., Li, L., Zhao, J., Liu, D., Yu, J., Wu, H., 2011. Benzo(a)pyrene-induced metabolic responses in Manila clam Ruditapes philippinarum by proton nuclear magnetic resonance $\left({ }^{1} \mathrm{H}\right.$ NMR) based metabolomics. Environ. Toxicol. Pharmacol. 32, 218-225. 